\title{
ORDER IDEALS, ANNIHILATOR IDEALS AND PATHOLOGICAL BEHAVIOR
}

\author{
E. GRAHAM EVANS AND PHILLIP GRIFFITH
}

\begin{abstract}
This article establishes a concrete relation between order ideals of minimal generators and annihilator ideals. For a regular local ring $R$ and ideal $I$ the authors construct an $R$-module $M$ with minimal generator having $I$ as order ideal. Further, it is shown that most variability in idealtheoretic behavior of such order ideals is exhibited by modules of projective dimension one. The authors "introduce" the concept of *-orthogonality and use their syzygy theorem to show constraints on the size and height of a *orthogonal set in a given finitely generated non-free module. The paper contains an application of the theory of order ideals to the binomial behavior of syzygy rank.
\end{abstract}

In article [12], we provided a summary update of results on order ideals associated to modules. These results and their application have been studied in detail since the late 1950's, beginning with the articles of Serre [17] and Bass [1]. The four central theorems outlined in [12] all focus on bounding heights (or grade) of order ideals, either from above or below. The applications resulting from these bounds extend from the theory of commutative algebra to that of algebraic geometry and cohomology of coherent sheaves (e.g., see the articles [12, Section 1], [15], [16]). Recently, Dutta [7] has shown the order ideal conjecture (open in mixed characteristic) for $k$ th syzygy modules of finite projective dimension implies the monomial conjecture in local algebra. Dutta's result places the theory of order ideals in a prominent place among the other homological conjectures, most of which have affirmative answers outside of mixed characteristic (see [4, Section III]). In the current article, we exhibit elementary constructions that demonstrate the wide range of behavior of such ideals, especially when the constraints of syzygy index and rank are relaxed. We make precise

2010 AMS Mathematics subject classification. Primary 13C99, 13D02.

Keywords and phrases. Regular local rings, modules, order ideals, syzygy rank and Betti numbers.

Received by the editors on June 21, 2014, and in revised form on October 13, 2014.

DOI: $10.1216 / J C A-2016-8-1-43$

Copyright (C)2016 Rocky Mountain Mathematics Consortium 
the notion of order ideal. For an $R$-module $E$ we use the notation $E^{*}=\operatorname{Hom}_{R}(E, R)$ to denote its $R$-dual.

Definition. Let $R$ be a ring, and let $E$ be an $R$-module. For $e \in E$, the associated ideal $O_{E}(e)=\left\{f(e) \mid f \in E^{*}\right\}$ is called the order ideal of $e$ in the module $E$. One can observe that the element $e$ defines a $R$-homomorphism $e: E^{*} \rightarrow R$ via the standard formula $e(f)=f(e)$. We note that image $(e)=O_{E}(e)$. If $E$ is a free $R$-module, then $O_{E}(e)$ is generated by the coordinate components of $e$ relative to a (any) $R$-basis for $E$. Of particular interest in the general theory is the case $E$ which represents a $k$ th module of syzygies, that is, inductively speaking, the case $E$ represents the module of relations on a $(k-1)$ st syzygy module $M$ for some (minimal) free resolution over the ring $R$. In this set-up, there is a prescribed generating set $m_{1}, \ldots, m_{g}$ for the $R$-module $M$ and a free $R$-module $R^{g}$ with the basis $\mathcal{E}_{1}, \ldots, \mathcal{E}_{g}$ such that $\mathcal{E}_{i} \mapsto m_{i}, 1 \leq i \leq g$, and such that $E=\operatorname{ker}\left(R^{g} \rightarrow M\right)$. It follows that an element $e \in E$ can be viewed as a vector $e=\left\langle\lambda_{1}, \ldots, \lambda_{g}\right\rangle \in R^{g}$ such that $\lambda_{1} m_{1}+\cdots+\lambda_{g} m_{g}=0$. Thus, $\operatorname{ht}_{R} O_{E}(e)$ can be interpreted to be a measure of the support for the relation defined by $e$.

In our original paper [8], monograph [10] and updated more recently in [12], we discuss the key results that show how syzygy index and rank serve to impose constraints on the behavior of order ideals, especially the behavior of order ideals for minimal generators (see also [9]). For example, if $R$ is a local ring containing a field and, if $E$ is a nonfree $k t h$ syzygy module over $R$ having finite projective dimension and rank $k$, then grade $O_{E}(e)=k$ for each minimal generator $e$ (that is, $e \in E-\mathbf{m} E$ where $\mathbf{m}$ denotes the maximal ideal of $R$ ).

In the current article, we pursue a theme mentioned in passing (see [12, page 405]) and examine more general and pathological behavior for order ideals of minimal generators. In Section 1 we begin with a few general facts and then draw a close connection between annihilator ideals and order ideals of minimal generators, e.g., see Proposition 1.3 and Remark 1.11. In particular, Remark 1.11 illustrates a key point that whatever pathology can be observed in the theory of order ideals for minimal generators can already be observed in modules $E$ having free presentations of the special form $0 \rightarrow R \rightarrow R^{t+1} \rightarrow E \rightarrow 0$. So, 
up to $R$-multiples, it suffices to study modules with a single defining relation.

The authors introduce the concept of $*$-orthogonality in Section 2 and use their syzygy theorem to show constraints on the size and height of a *-orthogonal set in a given finitely generated non-free module. In Section 2 we build on the example noted in [12, page 405] that shows the maximal ideal can occur multiple times as order ideal, even for modules of small rank. To facilitate this discussion, we introduce the notion of $*$-orthogonal sets to illustrate this phenomenon and its limitations. For example, we prove in Theorem 2.4 that a $*$-orthogonal set of $n$-elements having height $>k$ in a nonfree $k$ th syzygy module $E$ forces the inequality rank $E \geq n+k$. (Here, the underlying ring is regular local and contains a field.)

Section 3 contains an application of the theory of order ideals to the binomial behavior of syzygy rank. In Section 3, we exhibit a "factorization diagram" (Theorem 3.3) for suitable short exact sequences of syzygy modules. We apply the factorization result to conclude cases where syzygy modules have "appropriate" binomial rank. The diagram factorization is a byproduct of our work on order ideals of minimal generators, and provides a natural obstruction for maps between syzygy modules to be surjections. (See [5], [6], [11], [13], [14] for additional background on this material.)

Notation, terminology and restrictive assumptions. Our basic notation and terminology follows that of our monograph [9] and recent article [12]. The advanced book of Bruns and Herzog [4] is a valuable resource for unexplained facts about local rings and modules. Throughout this article, we assume (unless otherwise stated) the local ring is of the form

$$
R=\mathbf{k}\left[\left[X_{1}, \ldots, X_{n}\right]\right],
$$

where $\mathbf{k}$ is a field. A consequence of this assumption is that our syzygy theorem [10, Theorem 3.15] on ranks of $k$ th syzygy modules will always be valid when necessary for application in this setting. In addition, the pathological behavior discussed herein is not affected by our simplifying assumption. Finally, the symbol $\mathbf{m}$ denotes the unique maximal ideal of $R$, and of course, $\mathbf{k}$ denotes the residue field $\mathbf{k}=R / \mathbf{m}$. 
1. Relationship of order ideals to annihilator ideals. Our first observation asserts that the collection of order ideals obeys the following closure operations.

Lemma 1.1. The collection of order ideals of minimal generators over a fixed ring is closed under the algebraic operations of sum and intersection.

Proof. Closure for the sum operation follows readily from an application of the notion of direct sum. In the case of intersection $O_{E}(e) \cap O_{E^{\prime}}\left(e^{\prime}\right)$, where $e \in E-\mathbf{m} E$ and $e^{\prime} \in E^{\prime}-\mathbf{m} E^{\prime}$, we observe that $O_{E}(e) \cap O_{E^{\prime}}\left(e^{\prime}\right)=O_{E^{\prime \prime}}\left(e^{\prime \prime}\right)$, where $e^{\prime \prime}$ is represented by the coset $\langle\overline{e, o}\rangle=\left\langle\overline{o, e^{\prime}}\right\rangle \in\left(E \bigoplus E^{\prime}\right) / R\left\langle e,-e^{\prime}\right\rangle$, gives the desired result.

The following corollary will have an important application later in this section.

Corollary 1.2. Suppose that $I$ is a nonzero ideal in $R$ such that there is an injection

$$
\lambda: R / I \hookrightarrow \bigoplus M_{i}
$$

If $\lambda(1+I)=\left\langle m_{i}\right\rangle$ with $\operatorname{ann}_{R}\left(m_{i}\right)=O_{E}\left(e_{i}\right)$ for each $i$, then $\operatorname{ann}_{R}\left(\left\langle m_{i}\right\rangle_{i}\right)=\cap_{i} O_{E}\left(e_{i}\right)$ is also an order ideal for some minimal generator.

Proof. One has $\operatorname{ann}_{R}\left\langle m_{i}\right\rangle=\cap_{i} \operatorname{ann}_{R}\left(m_{i}\right)=\cap_{i} O_{E}\left(e_{i}\right)$ is an order ideal for some minimal generator from Lemma 1.1 .

Next, we draw a connection between order ideals and annihilator ideals-especially for particular torsion free $R$-modules of projective dimension one.

Proposition 1.3. Let $J=\left(x_{1}, \ldots, x_{t}\right)$ be a nonzero ideal, and let $x_{0} \in \mathbf{m}-J$. Form the short exact sequence $0 \rightarrow R \stackrel{\iota}{\rightarrow} R^{t+1} \stackrel{\eta}{\rightarrow} E \rightarrow 0$, where $\iota(1)=\left\langle x_{0}, x_{1}, \ldots, x_{t}\right\rangle$.

(i) If $e=\eta(\langle 1,0, \ldots, 0\rangle)$, then $\operatorname{ann}_{R}\left(x_{0}+J\right)=O_{E}(e)$ for the minimal generator $e$ of $E$.

(ii) If $\operatorname{ht}_{R}\left(x_{0}, x_{1}, \ldots, x_{t}\right) \geq k+1$, then $s y z_{R} E \geq k$. 
Proof. An $R$-homomorphism $f: R^{t+1} \rightarrow R$ induces an $R$-homomorphism $\bar{f}: E \rightarrow R$ precisely when $f\left(\left\langle x_{0}, \ldots, x_{t}\right\rangle\right)=0$. That is precisely when there is an $R$-relation $x_{0} r_{0}+x_{1} r_{1}+\cdots+x_{t} r_{t}=0$, where $r_{0}=f(\langle 1,0, \ldots, 0\rangle)$. So one has $f(e)=r \Leftrightarrow x_{0} r \in\left(x_{1}, \ldots, x_{t}\right)$.

For part (ii), we recall [8, Theorem 3.8] that $E$ becomes a $k$ th syzygy if and only if $E$ is free when localized at prime ideals of height $\leq k$.

Corollary 1.4. If $P=\left(a_{1}, \ldots, a_{t}\right)$ is a nonzero prime ideal of height $k<\operatorname{dim} R$, then $P=O_{E}(e)$ for a $k$ th syzygy module $E$ such that

(i) $e \in E-\mathbf{m} E$,

(ii) $p d E=1$,

(iii) $\operatorname{rank} E=t$.

Proof. Choose $a_{0} \in \mathbf{m}-P$, and note $\mathrm{ht}_{R}\left(a_{0}, P\right)=k+1$. Then apply the construction of Proposition 1.3.

Corollary 1.5. Let $P$ be as above. Then $P=O_{E}(e)$, where sy $z_{R} E=$ $k-1$ and

(i) $e \in E-\mathbf{m}, E$

(ii) $p d E=1$,

(iii) $\operatorname{rank} E=k$.

Proof. Let $x_{1}, \ldots, x_{k}$ be a maximal $R$-sequence in $P$. Then $P=$ $\operatorname{ann}_{R}\left(\left(x_{0},+\left(x_{1}, \ldots, x_{k}\right)\right)\right.$ for some $x_{0}$. Let $0 \rightarrow R \rightarrow R^{k+1} \rightarrow E \rightarrow 0$, where $1 \mapsto\left\langle x_{0}, x_{1}, \ldots, x_{k}\right\rangle$, and set $e=$ image of $\langle 1,0, \ldots, 0\rangle$ in $E$.

The next case deals with $\mathbf{m}$-primary ideals.

Corollary 1.6. Suppose $I$ is an $\mathbf{m}$-primary ideal and $x_{1}, \ldots, x_{d}$ is a maximal $R$-sequence in $I$. We assume that $\mathbf{x}=\left(x_{1}, \ldots, x_{d}\right) \neq \mathbf{m}$. Then $I=O_{E}(e)$ for some torsion free $R$-module $E$ and $e \in E-\mathbf{m} E$.

Proof. One has an injection $R / I \hookrightarrow \bigoplus^{h} R / \mathbf{x}$, since $R / \mathbf{x}$ is a selfinjective local ring. Suppose that $1+I=\left\langle\bar{a}_{1}, \ldots, \bar{a}_{h}\right\rangle$. Then the claim follows from Corollary 1.2 after one notes $I=\cap_{j}$ ann $\left(\bar{a}_{j}\right)$. 
Corollary 1.7. If depth $R / J>0$, then $J=O_{E}(e)$ for appropriate $E$ and $e \in E-\mathbf{m} E$.

Proof. There is $a_{0} \in \mathbf{m}-J$ such that $a_{0}$ is regular on $R / J$. So set $E=R^{t+1} / R v$ where $v=\left\langle a_{0}, \ldots, a_{t}\right\rangle$ and $J=\left(a_{1}, \ldots, a_{t}\right)$. Then ann $a_{0}+J=J$.

Theorem 1.8. Let $I$ be any nonzero ideal in $R$. Then $I=O_{E}(e)$ for appropriate $E$ and $e \in E-\mathbf{m} E$.

Proof. If depth $R / I>0$, we are finished by Corollary 1.7. If not, we can embed $R / I \hookrightarrow E(R / I)$, where $E(R / I)$ is the injective envelope of $R / I$. Then $E(R / I)=E_{0} \bigoplus E_{1}$ where Ass $E_{0}=\mathbf{m}$ and Ass $E_{1}$ contains only nonmaximal prime ideals. Let $C_{0}$ be the natural projection of $R / I$ in $E_{0}$ and likewise $C_{1}$ the natural projection of $R / I$ in $E_{1}$. Then, both $C_{0}$ and $C_{1}$ are cyclic with $\ell\left(C_{0}\right)<\infty$ and depth $C_{1}>0$. Therefore, the annihilators of $C_{0}$ and $C_{1}$ are order ideals of minimal generators for appropriate modules by Corollaries 1.6 and 1.7. Since we have $I=\operatorname{ann} C_{0} \cap \operatorname{ann} C_{1}$, we are finished by Corollary 1.2.

The final results of this section demonstrate that we often can be more efficient with respect to rank in our construction of modules having a minimal generator with prescribed order ideal. Due to the affirmative nature of the following two theorems we deviate from previous practice by allowing the local ring $(R, \mathbf{m})$ to denote any regular local ring-regardless of characteristic. The argument for Theorem 1.9 makes use of local cohomology as described in Brodmann and Sharp [2, Chapters 1-6].

Theorem 1.9. Let $(R, \mathbf{m})$ be a regular local ring, and let $I$ be an $R$ ideal of grade $\geq 3$. Then $I$ is the order ideal of a minimal generator for a rank 3 -module.

Proof. From our construction described in Proposition 1.3 one observes that it suffices to show there are elements $a, b, c, d$ in $\mathbf{m}$ such that $I=\operatorname{ann}_{R}(d+(a, b, c))$, that is, one needs to establish an $R$-injection $R / I \hookrightarrow R /(a, b, c)$.

To this end, let $E$ be the second syzygy module for $R / I$. We note $E$ is not a free $R$-module since pd $R / I \geq$ grade $I \geq 3$. Applying Bruns's 
theorem [4] we may construct a short exact sequence $O \rightarrow F \rightarrow E \rightarrow$ $E^{\prime} \rightarrow O$ for which $F$ is a free $R$-module and $E^{\prime}$ is the second syzygy module for a cyclic $R$-module of the form $R /(a, b, c)$. Since grade $I \geq 3$ one has that the local cohomology modules with respect to the ideal $I$ satisfy $H_{I}^{j}(R)=0$ for $j=0,1,2$. Therefore, one easily calculates the induced maps

$$
R / I \cong H_{I}^{0}(R / I) \cong H_{I}^{2}(E) \hookrightarrow H_{I}^{2}\left(E^{\prime}\right) \cong H_{I}^{0}(R /(a, b, c)) \subseteq R /(a, b, c) .
$$

Thus, there is the required injection $R / I \rightarrow R /(a, b, c)$, and the claim is proved.

Although we cannot establish the conclusion of Theorem 1.9 for all ideals $I$ having grade $I \geq 2$, we show in Theorem 1.10 that the conclusion of Theorem 1.9 holds for all prime ideals of grade $\geq 2$.

Theorem 1.10. Let $\operatorname{dim} R \geq 2$, and let $P$ be a prime ideal in $R$ with height $P \geq 2$. Then there is a torsion free $R$-module having rank 3 and minimal generator e such that $O_{E}(e)=P$.

Proof. When $P$ is the maximal ideal the result follows from our standard construction given in Proposition 1.3 and Bruns's theorem [3]. Namely, there is a three-generated ideal $(a, b, c)$ with $P$ associated to $R /(a, b, c)$. Therefore, one can form the short exact sequence

$$
O \longrightarrow R \stackrel{i}{\longrightarrow} R^{4} \longrightarrow E \longrightarrow O
$$

in which $i(1)=\langle d, a, b, c\rangle$ where $P=\operatorname{ann}[d+(a, b, c)]$. The general construction follows by repeating the identical construction over the local ring $R_{P}$. To insure that $E$ can be realized as an $R$-module one multiplies the vector $\langle d, a, b, c\rangle$ by a suitable $s \in R-P$ so that the adjusted vector $\langle s d, s a, s b, s c\rangle$ lies in $R^{4}$ and height $(s d, s a, s b, s c) \geq 2$. The affect of this operation does not change the $R$-annihilator of the coset $d+(a, b, c)$, i.e., $O_{E}(e)=P$ where $e$ represents the coset in $E$ for the vector $\langle 1,0,0,0\rangle \in R^{4}$. We are still left with the question: Can any grade $\geq 2$ ideal be the order ideal of a minimal generator in a torsion free $R$-module of rank 3 ?

Remark 1.11. While one may view the class of torsion free $R$-modules considered here to be rather special, we point out that the basic construction in Proposition 1.3 underlies most situations with respect 
to order ideals and their properties. To this end, let $E$ be a torsion free $R$-module, and let $e \in E-\mathbf{m} E$. We assume ht $O_{E}(e) \geq 2$ (if $O_{E}(e)$ is not principal, then $O_{E}(e)$ is always isomorphic to such an ideal.) Applying Bruns's theorem (see $[\mathbf{9}, 3.11]$ ) we may extend the set $\{e\}$ to a set $\left\{e=e_{0}, e_{1}, \ldots, e_{t-2}\right\}$ so that $\Sigma_{i} R e_{i}$ is a free submodule of $E$ and such that $E /\left(\Sigma_{i} R e_{i}\right)$ is isomorphic to an ideal of height $\geq 2$ (if the ideal in question were principal then $E$ would necessarily be a free module). Since an ideal of $R$ of height $\geq 2$ must contain a 2 -sequence, we enlarge our set $\left\{e_{0}, \ldots, e_{t-2}\right\}$ by two elements so that the enlarged set $\left\{e_{0}, \ldots, e_{t}\right\}$ has the following properties. For $W=\Sigma_{j=0}^{t} R e_{j}$

(a) There is a free presentation $0 \rightarrow R \rightarrow R^{t+1} \rightarrow W \rightarrow 0$, where $1 \mapsto\left\langle r_{0}, \ldots, r_{t}\right\rangle$ is the result of the relation

$$
r_{0} e_{0}+r_{1} e_{1}+\ldots+r_{t} e_{t}=0 .
$$

(b) All other relations on $\left\{e_{0}, \ldots, e_{t}\right\}$ are scalar multiples of $(*)$.

(c) $W^{*}=E^{*}$.

(d) $O_{W}\left(e_{0}\right)=O_{E}(e)=\operatorname{ann}\left(r_{0}+\left(r_{1}, \ldots, r_{t}\right)\right)$, i.e., $O_{E}(e)$ is an annihilator ideal modulo $\left(r_{1}, \ldots, r_{t}\right)$.

To see that (a)-(d) hold one concludes from the construction of $W$ that $t=\operatorname{rank}_{R} E, W$ is generated by $1+\operatorname{rank} W=1+t$ elements and that $E / W$ has no support in codimension one. Also, part (d) is related to Proposition 1.3 (i).

Thus, any torsion free module $E$ contains a copy of such a module " $W$ " for each of its generators.

2. *-Orthogonal sets, rank and order ideals for syzygy modules of projective dimension one. We begin with a discussion of *-orthogonal sets and their consequences in the setting of torsion free modules.

Definition 2.1. Let $(R, \mathbf{m})$ be a local domain, and let $E$ be a torsion free $R$-module. A set $\left\{e_{i}\right\}$ in $E$ is called a $*$-orthogonal set, provided: for each $i$, the order ideal $O_{E}\left(e_{i}\right)$ is generated by $f\left(e_{i}\right)$ where $f \in E^{*}$ and $f\left(e_{j}\right)=0$ for $j \neq i$. We say the set $\left\{e_{i}\right\}$ is $*$-orthogonal of height $\geq k$ provided ht $O_{E}\left(e_{i}\right) \geq k$ for each $i$. 
Remark 2.2. Choosing an element $f_{i}: \in E^{*}$ for each $i$ such that $f_{i}\left(e_{i}\right) \neq 0$ while $f_{i}\left(e_{j}\right)=0$ for $i \neq j$ gives rise to an $R$-bilinear form on $E$ defined by the formula: $\left\langle e, e^{\prime}\right\rangle=\Sigma_{i} f_{i}(e) f_{i}\left(e^{\prime}\right)$ for which the set $\left\{e_{1}, \ldots, e_{n}\right\}$ in the definition (2.1) is an orthogonal set in the usual sense.

Lemma 2.3. Suppose $E$ is a kth syzygy module having a *-orthogonal set $\left\{e_{i}\right\}$ of height $>k$. Then $E / F$ is a kth syzygy module and $F=\Sigma_{i} \operatorname{Re}_{i}$ is a free submodule.

Proof. The argument here is a direct consequence of [10, Lemma 3.10] and induction on $n$.

The following statement amounts to a restatement of our syzygy theorem [10, Theorem 3.15] in the context of $*$-orthogonal sets.

Theorem 2.4. Let $E$ be a nonfree $k$ th syzygy module. If $\left\{e_{1}, \ldots, e_{n}\right\}$ is a $*$-orthogonal set in $E$ of height $>k$, then $\operatorname{rank} E \geq k+n$.

Proof. The definition of $*$-orthogonal set together with induction on $n$ guarantees the short exact sequence

$$
0 \longrightarrow \Sigma_{i} \operatorname{Re}_{i} \longrightarrow E \longrightarrow E / \Sigma_{i} \operatorname{Re}_{i} \longrightarrow 0
$$

is split exact locally in codimension $\leq k$. Thus, the factor module $E / \Sigma_{i} \operatorname{Re}_{i}$ will necessarily be a $k$ th syzygy module when $E$ is a $k$ th syzygy module.

We remark that a basis $\left\{e_{1}, \ldots, e_{n}\right\}$ for a free module $F=\bigoplus_{i} \operatorname{Re}_{i}$ is certainly a $*$-orthogonal set as a result of the notion of "dual basis." The following corollary indicates a module with a $*$-orthogonal set is necessarily free should the $*$-orthogonal set be too large in relation to the rank of the module.

Corollary 2.5. If the kth syzygy $E$ has a $*$-orthogonal set $\left\{e_{1}, \ldots, e_{n}\right\}$ of height $>k$ such that $n>\operatorname{rank} E-k$, then $E$ is a free $R$-module.

In particular, if $E$ is a reflexive module (i.e., $E$ is a second syzygy) then $n>\operatorname{rank} E-2$ implies that $E$ is a free module. This form of 
Corollary 3.5 will be used later in the current section. In this context the following observation will prove useful.

Lemma 2.6. Let $E$ be a torsion free $R$-module, and let $\left\{e_{1}, \ldots, e_{n}\right\}$ be $a$ *-orthogonal set in $E$ of height $\geq 3$. Then $0 \rightarrow F \rightarrow E^{* *} \rightarrow$ $(E / F)^{* *} \rightarrow 0$ is exact, where $F=\Sigma \operatorname{Re}_{i}$.

Proof. From the short exact sequence $0 \rightarrow F \rightarrow E \rightarrow E / F \rightarrow 0$ we obtain the induced commutative diagram of

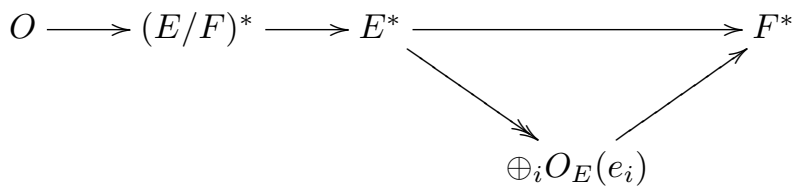

which further induces the commutative diagram

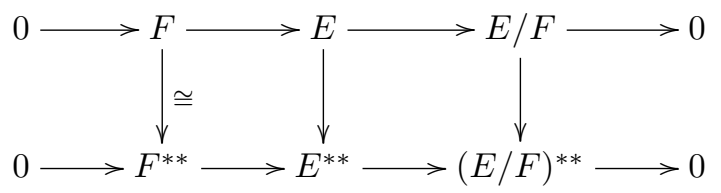

The map $E^{* *} \rightarrow(E / F)^{* *}$ is surjective because $\operatorname{Ext}_{R}^{1}\left(O_{E}\left(e_{i}\right), R\right)=0$, for $1 \leq i \leq n$, since height $O_{E}\left(e_{i}\right) \geq 3$ for each $i$.

Corollary 2.7 (Notation as above). The second dual $E^{* *}$ is free if and only if $(E / F)^{*}$ is free.

Our next goal is to provide an affirmative answer to our question [12, page 14] regarding generating sets having multiple members whose order ideals are the maximal ideal. As it turns out, the members $e$ of the generating set having the property $O_{E}(e)=\mathbf{m}$ are members of a *-orthogonal set of generators. We describe our construction here.

Following the setup in [12, page 405] let $(a, b, c)$ denote a threegenerated ideal having the following properties:

(*) The vector space dimension of the $\mathbf{k}$-socle of $R /(a, b, c)$ is $n$.

$(* *)$ The second syzygy of $R /(a, b, c)$ is nonfree of rank 2 . 
Let $m_{1}, \ldots, m_{n}$ be elements of $R$ whose cosets modulo $(a, b, c)$ provide a $\mathbf{k}$-basis for the $\mathbf{k}$-socle of $R /(a, b, c)$. That is, the annihilator of each coset $m_{i}+(a, b, c)$ is exactly $\mathbf{m}$. We let $E$ be defined by the minimal free presentation $0 \rightarrow R \rightarrow R^{n+3} \rightarrow E \rightarrow 0$ where $1 \rightarrow\left\langle m_{1}, \ldots, m_{n}, a, b, c\right\rangle$. Let $e_{1}, \ldots, e_{n}$ be the corresponding first $n$-standard basis vectors in $R^{n+3}$ whose images in $E$ are denoted by $\bar{e}_{1}, \ldots, \bar{e}_{n}$, respectively. As argued in [12, page 14], we have $O_{E}\left(e_{i}\right)=\mathbf{m}$, for $1 \leq i \leq n$. The set $\left\{\bar{e}_{1}, \ldots, \bar{e}_{n}\right\}$ is easily shown to be a $*$-orthogonal set in $E$ having $n=\operatorname{rank} E-2$ elements. The question asked in [12, page 405] is: can we find a larger such set? The answer is given in our next result and is achieved through a more subtle argument than say that of Theorem 2.4.

Theorem 2.8. Let $E$ be a torsion free $R$-module such that $E^{*}$ is not a free module, and let $\left\{e_{1}, \ldots, e_{n}\right\}$ be a $*$-orthogonal set in $E$ of height $\geq 3$. Then $n \leq \operatorname{rank} E-2$.

Proof. Consider the case where $n=\operatorname{rank} E-1$, and let $F$ be the free submodule generated by $e_{1}, \ldots, e_{n-1}$. Then the rank 2 torsion free factor module $N=E / F$ has an element $\bar{e}_{n}$ having order ideal $J$ of height $\geq 3$. Therefore, we obtain a short exact sequence

$$
0 \longrightarrow R \longrightarrow N \longrightarrow N / R \longrightarrow 0, \quad \text { where } 1 \longmapsto \bar{e}_{n},
$$

and where $N / R$ is isomorphic to an ideal of $R$. Dualizing (*) with respect to $R$ gives an exact sequence

$$
0 \longrightarrow(N / R)^{*} \longrightarrow N^{*} \longrightarrow R^{*} \longrightarrow \operatorname{Ext}_{R}^{1}(N / R, R) .
$$

The image of $N^{*}$ in $R^{*} \cong R$ is isomorphic to $J$ and $(N / R)^{*} \cong R$ since $R$ is a $U F D$. From here, one sees that the left hand portion of the dual sequence $0 \rightarrow R \rightarrow N^{*} \rightarrow J \rightarrow 0$ is necessarily split exact since height $J=$ grade $J \geq 3$ implies $\operatorname{Ext}_{R}^{1}(J, R) \cong \operatorname{Ext}_{R}^{2}(R / J, R)=0$.

Thus, $N^{*} \cong R \bigoplus J$ is free since $J$ is a reflexive ideal and $R$ is a $U F D$. Noting that $N^{*}=(E / F)^{*}$ and $N^{* *}=(E / F)^{* *}$ are free $R$-modules we may invoke Corollary 2.7 (see also Lemma 2.6) to conclude $E^{*}$ must be a free $R$-module as well, a fact that contradicts the hypothesis above.

Remark. We note the condition in Theorem 2.8 "height $\geq 3$ " is necessary. If $R=k[[x, y, z]]$ and $N=R^{3} /\left\langle x^{2}, y^{2}, x y\right\rangle$, then the order 
ideal of the image of $\langle 0,0,1\rangle$ in $N$ is $(x, y), \operatorname{rank} N=2$ and $N^{*}$ is not a free module.

We recall more facts concerning our example [12, page 14] cited earlier in this section. From the description surrounding our example one may construct the commutative diagram

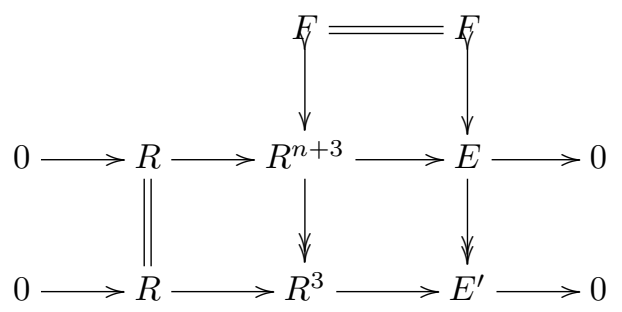

where $1 \mapsto\langle a, b, c\rangle$ defines the left hand map of the second row. It follows that $\left(E^{\prime}\right)^{*}$ is the second syzygy for the cyclic module $R /(a, b, c)$. By assumption, $R /(a, b, c)$ was chosen so that the second syzygy module for $R /(a, b, c)$ was not free. The assumption was made possible by Bruns's theorem (see [10, Corollary 3.13] or the original paper [3]). Thus, the $*$-orthogonal set in which each member had order ideal $\mathbf{m}$ cannot be extended to a larger one having the same property.

When one deletes the "*-orthogonal" requirement on the set of minimal generators that have order ideal equal to $\mathbf{m}$, then one can construct modules in which an entire minimal generating set has this property. By way of example, we set $R=\mathbf{k}[[x, y, z, w]]$ where the coefficient field has characteristic different from 2 . We define the four-generated module $E$ of rank 3 via the minimal free presentation $0 \rightarrow R \rightarrow R^{4} \rightarrow E \rightarrow 0$ where $1 \mapsto\langle z w, x z, y w, x y\rangle$, and let $\bar{e}_{1}, \bar{e}_{2}, \bar{e}_{3}, \bar{e}_{4}$ represent the images in $E$ of the usual standard basis elements $e_{1}, e_{2}, e_{3}, e_{4}$, respectively, for $R^{4}$. Then the collection $\bar{e}_{1}+$ $\bar{e}_{4}, \bar{e}_{1}-\bar{e}_{4}, \bar{e}_{2}+\bar{e}_{3}, \bar{e}_{2}-\bar{e}_{3}$ is the desired minimal generating set. We calculate the order ideal for $\bar{e}_{1}+\bar{e}_{4}$ from the relation

$$
z w\left(\bar{e}_{1}+\bar{e}_{4}\right)+x z \bar{e}_{2}+y w \bar{e}_{3}+(x y-z w) \bar{e}_{4}=0
$$

and rely on symmetry of relation and argument for the remaining three. (Note: $z w \bar{e}_{1}+x z \bar{e}_{2}+y w \bar{e}_{3}+x y \bar{e}_{4}=0$ is given as a result of the free presentation.) A straightforward calculation shows $\mathbf{m}(z w) \subseteq$ $(x z, y w, x y-z w)$, that is, $\mathbf{m}=\operatorname{ann}(z w)$ modulo the ideal $(x z, y w, x y$ - $z w$ ). From Proposition 1.3 (i), we get $\mathbf{m}=O_{E}\left(\bar{e}_{1}+\bar{e}_{4}\right)$. Similar 
arguments work for the remaining minimal generators $\bar{e}_{1}-\bar{e}_{4}, \bar{e}_{2}+$ $\bar{e}_{3}, \bar{e}_{2}-\bar{e}_{3}$.

Since the ideal $(z w, x z, y w, x y)=(y, z) \cap(x, w)$ has height 2 , we note that the module $E$ is a first syzygy module (i.e., $E$ is torsion free) but not a second syzygy module. Finally we remark that the order ideals for the minimal generating set $\bar{e}_{1}, \bar{e}_{2}, \bar{e}_{3}, \bar{e}_{4}$ are the collection of height 2 prime ideals $(x, y),(y, w),(x, z)$ and $(z, w)$, respectively.

In closing, we make two additional observations about $*$-orthogonal sets. Although the center of our attention in this article concerns order ideals of minimal generators, on occasion we have dropped that assumption when discussing $*$-orthogonal sets, e.g., see Theorem 2.4. However, if a proposed order ideal $O_{E}(e)$ is not contained in $\mathbf{m}^{2}$ when $E$ has no free summands (e.g., say $O_{E}(e) \nsubseteq \mathbf{m}^{2}$ ), then $e$ is necessarily a minimal generator. Moreover, if $E^{*}$ has no free summands then $O_{E}(e) \nsubseteq \mathbf{m}^{2}$ implies $e$ is also a minimal generator of $E^{* *}$. Our final observation of this section concerns $*$-orthogonal sets and free bases.

Proposition 2.9. Let $E$ be a torsion free module of rank $n$, and suppose $e_{1}, \ldots, e_{n}$ is a $*$-orthogonal set of height $\geq 2$. Then $E$ is a free module with bases $e_{1}, \ldots, e_{n}$.

Proof. Our conclusion follows directly from Theorem 2.4. Since the factor module $E / F$ must satisfy the Serre condition $\left(S_{1}\right)$, i.e., $E / F$ is torsion free, it must be that $E / F=0$.

\section{Application of the theory of order ideals to binomial} behavior of syzygy rank. Given an $R$-homomorphism $\varphi: M \rightarrow N$, we refer to $\varphi$ as a proper map provided the induced $\operatorname{map} \bar{\varphi}: M / \mathbf{m} M \rightarrow$ $N / \mathbf{m} N$ is a nonzero homomorphism; that is, $\varphi$ is proper provided $1 \otimes \varphi: \mathbf{k} \otimes M \rightarrow \mathbf{k} \otimes N$ is nonzero. More simply put, a map $\varphi: M \rightarrow N$ is proper provided there is a minimal generator $m \in M$ that is mapped by $\varphi$ to a minimal generator $n \in N$. So $\varphi(m)=n \in N-\mathbf{m} N$. The central questions addressed here go as follows: given a short exact sequence $0 \rightarrow \mathbf{k} \rightarrow T \rightarrow T^{\prime} \rightarrow 0$, how can one decide whether the induced maps of syzygy modules are proper or not? And, if one can ascertain the answer, how does this information impact ranks of syzygy modules? Our initial observation begins with the following obvious fact about order ideals. 
Lemma 3.1. Let $\varphi: M \rightarrow N$ be a homomorphism of $R$-modules such that $\varphi(m)=n$. Then $O_{N}(n) \subseteq O_{M}(m)$.

The next step is to be able to compute order ideals for syzygy modules. To this end, we state the following proposition.

Proposition 3.2. Let $0 \rightarrow E \rightarrow F \rightarrow M \rightarrow 0$ be short exact in which the middle term $F$ is a free $R$-module.

(a) If $e \in E$, then $O_{F}(e) \subseteq O_{E}(e)$ and $O_{F}(e)$ is completely determined by any coordinate representation of $e$ in $F$.

(b) If $\operatorname{Ext}_{R}^{1}(M, R)=0$, then equality $O_{F}(e)=O_{E}(e)$ holds.

Proof. Part (a) follows directly from (3.1) and the fact $O_{F}(e)$ is generated by the coordinates of $e$ relative to (any) $R$-basis for $F$.

Part (b) follows from the "dual exactness" $0 \rightarrow M^{*} \rightarrow F^{*} \rightarrow E^{*} \rightarrow 0$ that results when $\operatorname{Ext}_{R}^{1}(M, R)=0$. Note every $R$-homomorphism $E \rightarrow R$ extends to $F \rightarrow R$ in this case. So $O_{F}(e)=O_{E}(e)$.

The above simple observations can be very useful in computing order ideals for syzygy modules in the following context. Let $F_{\bullet} \rightarrow T$ be a minimal free $R$-resolution of a module $T$ having finite length. Let $E_{k}=$ $\operatorname{Syz}_{k}(T)$ for each index $k$ in this free resolution. Then $O_{E}(e)=O_{F}(e)$, for $e \in E_{k}$ and $1 \leq k \leq \operatorname{dim} R-1$. Moreover, if $e \in E_{k}-\mathbf{m} E_{k}$, then $O_{F}(e)$ can be determined from its basis representation in $F_{k-1}$ (we are assuming here that matrix representations for the differential maps in $F_{\bullet}$ are given.) For example, if $T=\mathbf{k}$, then all of the order ideals of minimal generators in the syzygy modules are linear in the sense each is minimally generated by elements in $\mathbf{m}-\mathbf{m}^{2}$. In this special case, we use the notation " $W_{k}$ " to denote the $k$ th syzygy module (unique up to isomorphism) for the residue field $\mathbf{k}$. A short exact sequence $0 \rightarrow T^{\prime} \rightarrow T \rightarrow T^{\prime \prime} \rightarrow 0$ of $R$-modules induces via the horseshoe construction a short exact sequence of free resolutions which in turn induces a finite sequence of short exact sequences of syzygy modules $0 \rightarrow E_{k}^{\prime} \rightarrow L_{k} \bigoplus E_{k} \rightarrow E_{k}^{\prime \prime} \rightarrow 0$ where the free $R$-modules " $L_{k}$ " are necessary since the middle free resolution in the horseshoe lemma construction may not be minimal. Under appropriate conditions, we obtain a useful factorization diagram in this setting. A version of this result was informally discussed in our article [12, Section 4]. 
Theorem 3.3. Let $0 \rightarrow E^{\prime} \rightarrow L \bigoplus E \rightarrow E^{\prime \prime} \rightarrow 0$ be a short exact sequence of $R$-modules in which $L$ is $R$-free.

(a) If the induced map $E \rightarrow E^{\prime \prime}$ is not proper, then there is an induced commutative diagram

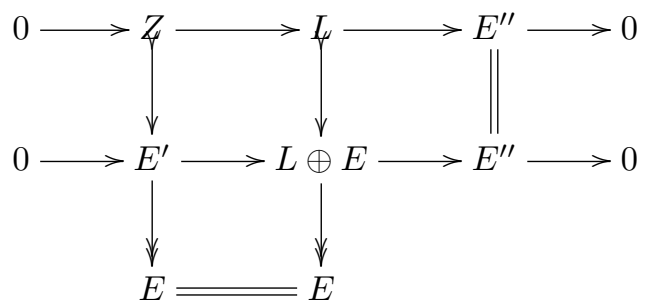

in which the middle column is split exact and the first column is exact.

(b) If $E^{\prime}$ is without nontrivial free $R$-summands, then the first row is a minimal free presentation of $E^{\prime \prime}$.

Proof.

(a) Under the standing hypothesis, no minimal generator of $E$ can map to a minimal generator of $E^{\prime \prime}$; thus Image $\left(E \rightarrow E^{\prime \prime}\right) \subseteq \mathbf{m} E^{\prime \prime}$. It follows that the induced map $L \rightarrow E^{\prime \prime}$ is necessarily surjective and so the commutative diagram as described above follows as well.

(b) The upper left square of the commutative diagram in part (a) shows that any shared free summand of $Z \rightarrow L$ will likewise produce a shared free summand of $Z \rightarrow E^{\prime}$ having the same rank. Thus, claim (b) follows.

Corollary 3.4. We suppose that $0 \rightarrow \mathbf{k} \rightarrow T \rightarrow T^{\prime} \rightarrow 0$ is nonsplit, $E_{k}^{\prime}=S y z_{k}\left(T^{\prime}\right)$ has a generating set for which no order ideal contains a linear $R$-sequence of length $k-1$, and $E_{k}=S y z_{k}(T)$ has no nontrivial free summands. Then, for $k \geq 2$, there is a short exact sequence $0 \rightarrow W_{k} \rightarrow E_{k} \rightarrow E_{k}^{\prime} \rightarrow 0$.

Remark 3.5. The assumption that the short exact sequence $0 \rightarrow \mathbf{k} \rightarrow$ $T \rightarrow T^{\prime} \rightarrow 0$ is not split is natural here since the split exact case is easily analyzed. 
Proof. Since $0 \rightarrow \mathbf{k} \rightarrow T \rightarrow T^{\prime} \rightarrow 0$ is nonsplit, then $\mathbf{k} \subseteq \mathbf{m} T$ which implies there is a short exact sequence of syzygy modules $(*) 0 \rightarrow E_{1} \rightarrow$ $E_{1}{ }^{\prime} \rightarrow \mathbf{k} \rightarrow 0$. Applying the usual horseshoe construction to obtain simultaneous free $R$-resolutions for short exact sequence $(*)$ one has short exact sequences for $k \geq 2,0 \rightarrow E_{k} \rightarrow L_{k} \oplus E_{k}{ }^{\prime} \rightarrow W_{k-1} \rightarrow 0$, where $L_{k}$ is a free $R$-module. If there is a $k \geq 2$ so that $E_{k}{ }^{\prime}$ has a generating set for which no element has an order ideal containing a linear $R$-sequence of length $k-1$, then those minimal generators must map to $\mathbf{m} W_{k-1}$ which implies Image $\left(E_{k}{ }^{\prime} \rightarrow W_{k-1}\right) \subseteq \mathbf{m} W_{k-1}$. Therefore, applying our factorization theorem, gives a short exact sequence $0 \rightarrow W_{k} \rightarrow E_{k} \rightarrow E_{k}^{\prime} \rightarrow 0$.

The most obvious situation to apply Corollary 3.4 is the case for which $\ell(T)<\infty$. For $1 \leq k<\operatorname{dim} R$, the $k$ th syzygy module $E_{k}$ will not have nontrivial free $R$-summands. Working under the assumption that we know the matrix representations for the differential $d . '$ in the $R$-free resolution $F_{\bullet}^{\prime} \rightarrow T^{\prime}$, we may apply (3.4) in instances where the entries for $d_{k}^{\prime}$ lie in $\mathbf{m}^{2}$.

Example 3.6. Let $R=\mathbf{k}\left[\left[x_{1}, x_{2}, x_{3}, x_{4}, x_{5}\right]\right]$, and set $J=x_{1}^{2} \mathbf{m}+$ $\left(x_{2}^{2}, x_{3}^{2}, x_{4}{ }^{2}, x_{5}{ }^{2}\right)$. Note the containment $J \subset\left(x_{1}^{2}, x_{2}^{2}, x_{3}{ }^{2}, x_{4}{ }^{2}, x_{5}{ }^{2}\right)=$ $I$ is strict and $0 \rightarrow I / J \rightarrow R / J \rightarrow R / I \rightarrow 0$ is exact with $I / J \cong \mathbf{k}$. Since the differentials for the $R$-free resolution for $R / I$ can be defined over $k\left[\left[x_{1}{ }^{2}, x_{2}{ }^{2}, x_{3}{ }^{2}, x_{4}{ }^{2}, x_{5}{ }^{2}\right]\right]$, one may apply the conclusion of Corollary 3.4 to obtain that $\operatorname{rank} \operatorname{Syz}_{k}(R / J)=2\left(\begin{array}{c}4 \\ k-1\end{array}\right)$ for $k=2,3,4$.

For more discussion on the binomial behavior of syzygy rank and Betti numbers for modules of finite length, one may consult the articles of Herzog and Kühl [14], Evans and Griffith [11], Charalambous [5] and Charalambous and Evans [6].

Acknowledgments. The authors thank the referee for a careful reading of their manuscript that produced essential corrections and sharper arguments.

\section{REFERENCES}

1. H. Bass, K-Theory and stable algebra, Publ. Math. 22, IHES, Paris, 1964.

2. M.P. Brodmann and R.Y. Sharp, Local cohomology, Cambr. Stud. Adv. Math. 60, Cambridge University Press, Cambridge, 1998. 
3. W. Bruns, "Jede" endliche freie Auflösung is freie Auflösung eines von drei Elementen erzeugten Ideals, J. Alg. 39 (1976), 429-439.

4. W. Bruns and J. Herzog, Cohen-Macauley rings, Cambr. Stud. Adv. Math. 39, Cambridge University Press, Cambridge, 1993.

5. H. Charalambous, Betti numbers of multigraded modules, J. Alg. 137 (1991), 491-500.

6. H. Charalambous and E.G. Evans, A deformation theory approach to Betti numbers of finite length modules, J. Alg. 143 (1991), 246-251.

7. S. Dutta, The monomial conjecture and order ideals, J. Alg. 383 (2013), $232-241$.

8. E.G. Evans and P. Griffith, The syzygy problem, Ann. Math. 114 (1981), 323-333.

9. (1982), 375-378.

10. , Syzygies, Lond. Math. Soc. Lect. Note Ser. 106, Cambridge University Press, Cambridge, 1985.

11. Binomial behavior of Betti numbers for modules of finite length, Pac. Math. J. 133 (1988), 267-276.

12. A A brief history of order ideals, in Commutative algebra: Expository papers dedicated to David Eisenbud on the occasion of his 65th birthday 8 (2013), page 31 .

13. P. Griffith and A. Seceleanu, Syzygy theorems via comparison of order ideals on a hypersurface, J. Pure Appl. Alg. 216 (2012), 468-479.

14. J. Herzog and M. Kühl, On the Betti numbers of finite pure and linear resolutions, Comm. Alg. 12 (1984), 1627-1646.

15. R. Lazarsfeld and M. Popa, Derviation complex, BGG correspondence, and numerical inequalities for compact Kähler manifolds, Invent. 182 (2010), 605-633.

16. A. Pareschi and M. Popa, Strong generic vanishing and a higherdimensional Castelnuovo-de Franchis inequality, Duke Math. J. 150 (2009), 269285 .

17. J.-P. Serre, Sur les modules projectifs. Seminaire Dubriel-Piscot, 13, Paris, $1960-61$.

611 Harding Drive, Urbana, IL 61801

Email address: geevans64@gmail.com

2705 Holcomb Dr., Urbana, IL 61802

Email address: pgriffit@illinois.edu 